\title{
Thermostatistics of overdamped motion of interacting particles
}

\author{
J. S. Andrade Jr. ${ }^{1,3}$, G. F. T. da Silva ${ }^{1}$, A. A. Moreira ${ }^{1}$, F. D. Nobre ${ }^{2,3}$, E. M. F. Curado ${ }^{2,3}$ \\ ${ }^{1}$ Departamento de Física, Universidade Federal do Ceará, 60451-970 Fortaleza, Ceará, Brazil \\ ${ }^{2}$ Centro Brasileiro de Pesquisas Físicas, Rua Xavier Sigaud 150, 22290-180, Rio de Janeiro-RJ, Brazil \\ ${ }^{3}$ National Institute of Science and Technology for Complex Systems, \\ Rua Xavier Sigaud 150, 22290-180, Rio de Janeiro-RJ, Brazil
}

(Dated: October 30, 2018)

\begin{abstract}
We show through a nonlinear Fokker-Planck formalism, and confirm by molecular dynamics simulations, that the overdamped motion of interacting particles at $T=0$, where $T$ is the temperature of a thermal bath connected to the system, can be directly associated with Tsallis thermostatistics. For sufficiently high values of $T$, the distribution of particles becomes Gaussian, so that the classical Boltzmann-Gibbs behavior is recovered. For intermediate temperatures of the thermal bath, the system displays a mixed behaviour that follows a novel type of thermostatistics, where the entropy is given by a linear combination of Tsallis and Boltzmann-Gibbs entropies.
\end{abstract}

PACS numbers: 05.45.-a, 05.40.Fb, 05.10.Gg, 05.20.-y

Nonlinear Fokker-Planck equations (FPE's) 1] are frequently employed to represent macroscopically physical and chemical systems displaying anomalous diffusion behavior [2]. Scientifically and technologically important examples of such systems include, among others, the flow through porous media 3], the dynamics of surface growth [4], the diffusion of polymer-like breakable micelles [5], and the dynamics of interacting vortexes in disordered superconductors [6 8]. A typical nonlinear FPE may be written in the general form [1, 9],

$$
\frac{\partial P}{\partial t}=-\frac{\partial[A(x) \Psi(P)]}{\partial x}+\frac{\partial}{\partial x}\left[\Omega(P) \frac{\partial P}{\partial x}\right]
$$

where the external force $A(x)$ is associated with a potential $\phi(x)[A(x)=-d \phi(x) / d x]$, and the analyticity of the potential as well as the integrability of the force are assumed to hold in all space. The functionals $\Psi[P(x, t)]$ and $\Omega[P(x, t)]$ satisfy requirements of positiveness, integrability, and differentiability with respect to $P(x, t)$ [9]. Moreover, in order to preserve the probability normalization for all times, one should impose the probability distribution, together with its first derivative, as well as the product $A(x) \Psi[P(x, t)]$, to be zero in the limits $x \rightarrow \pm \infty$.

An important result associated with nonlinear FPE's is the H-theorem and its generalizations [1, 9 13]. In the case of a system subjected to an external potential, the H-theorem leads to a well-defined sign for the time derivative of the free-energy functional,

$$
F=U-\gamma S ; \quad U=\int_{-\infty}^{\infty} d x \phi(x) P(x, t)
$$

where $\gamma$ represents a positive Lagrange multiplier and the entropy may be considered in a very general form as,

$$
S[P]=\int_{-\infty}^{\infty} g[P(x, t)] d x ; g(0)=g(1)=0 ; \frac{d^{2} g}{d P^{2}} \leq 0,
$$

with the condition that $g[P(x, t)]$ should be at least twice differentiable. Considering the FPE (1), for $d F / d t \leq 0$
[9, 13], we obtain,

$$
-\gamma \frac{d^{2} g[P]}{d P^{2}}=\frac{\Omega[P]}{\Psi[P]} .
$$

A relevant outcome of Eq. (44) is that the ratio $\Omega[P] / \Psi[P]$ determines an entire class of FPE's associated with a single entropic form [9, 13]. Here we are interested on the following type of nonlinear FPE [14, 15]:

$$
\frac{\partial P}{\partial t}=-\frac{\partial[A(x) P]}{\partial x}+D \nu \frac{\partial}{\partial x}\left[P^{\nu-1} \frac{\partial P}{\partial x}\right],
$$

where $D$ is a constant, $\nu$ is a real number and the functionals in Eq. (10) correspond to $\Psi[P(x, t)]=P(x, t)$ and $\Omega[P(x, t)]=D \nu[P(x, t)]^{\nu-1}$. By substituting these quantities in Eq. (4), integrating and using the conditions (3), one obtains Tsallis entropy [9, 16, 17], for which,

$$
g[P]=k \frac{[P(x, t)]^{\nu}-P(x, t)}{1-\nu},
$$

where $k \equiv D / \gamma$. Considering an external force $A(x)=$ $-\alpha x(\alpha \geq 0)$, the solution for Eq. (5) with initial condition $P(x, 0)=\delta(x)$ is given by the distribution,

$$
P(x, t)=B(t)\left[1+\beta(t)(1-\nu) x^{2}\right]_{+}^{1 /(\nu-1)},
$$

where $[y]_{+}=y$, for $y>0$ and zero otherwise. The timedependent parameters $B(t)$ and $\beta(t)$ are defined in such a way as to preserve the norm and the form of the distribution for all times [14, 15, 18]. Equation (7) corresponds exactly to the distribution obtained through extremization of Tsallis entropy Eq. (6), for the energy defined as in Eq. (2), and under standard constraints of probability normalization. It is important to notice that, by substituting $\nu=2-q$ in Eq. (7), one recovers precisely the usual distribution of nonextensive statistical mechanics, known as $q$-Gaussian, as obtained through a more general definition for the internal energy [17]. 
Additionally, the way in which the system evolves dynamically, as well as its stationary state, provide distinctive signatures of anomalous diffusion behavior. For small times $t \ll 1$, it is possible to show that the diffusion propagation front $x(t)$ dictated by Eq. (5) advances as $\langle x(t)\rangle \propto t^{2 /(\nu+1)}$ [13]. In the limit $t \rightarrow \infty$, the system approaches the stationary state given by,

$$
P_{\mathrm{st}}(x)=B^{*}\left[1-\beta^{*} x^{2}\right]_{+}^{1 /(\nu-1)}
$$

depending on the initial time $t_{0}, \beta^{*}=\beta\left(t_{0}\right)\left[B^{*} / B\left(t_{0}\right)\right]^{2}$, and $B^{*}=\left[\alpha B\left(t_{0}\right)^{2} / 2 D \nu \beta\left(t_{0}\right)\right]^{1 /(1+\nu)}$. One should stress that this form of stationary distribution holds for any confining potential $\phi(x)$, by simply replacing in Eq. (8) the term $x^{2}$ with $\phi(x)[14]$.

Next we show that the microscopic behavior of a system of interacting particles undergoing overdamped motion is fully compatible with a continuum nonlinear diffusion equation. Moreover, we find that such a continuum formulation for a highly dissipative system corresponds to the FPE (5) with $\nu=2$. We start by considering the equation of motion for a particle $i$ in a system of $N$ overdamped particles,

$$
\mu \vec{v}_{i}=\sum_{j \neq i} \vec{J}\left(\vec{r}_{i}-\vec{r}_{j}\right)+\vec{F}^{e}\left(\vec{r}_{i}\right)+\eta\left(\vec{r}_{i}, t\right)
$$

where $\vec{v}_{i}$ is the velocity of the $i$ th particle, $\mu$ is the effective viscosity of the medium, the first term on the right accounts for the interactions among particles, $\vec{F}^{e}\left(\vec{r}_{i}\right)$ represents an external force, and $\eta$ corresponds to an uncorrelated thermal noise with zero mean and variance $\left\langle\eta^{2}\right\rangle=k_{B} T / \mu$. Here we consider a short-range repulsive particle-particle interaction in the form, $\vec{J}(\vec{r}) \equiv$ $G(|\vec{r}| / \lambda) \hat{r}$, where $\hat{r}$ is the unit vector along the axis connecting each pair of particles, and $\lambda$ is a characteristic length of the short-range pairwise interaction.

To obtain a continuum description of this system [6], we perform a coarse graining of Eq. (9), starting from the Fokker-Planck equation for the probability distribution of the particle coordinates $\mathcal{P}\left(\vec{r}_{1}, \ldots, \vec{r}_{N}, t\right)$,

$$
\mu \frac{\partial \mathcal{P}}{\partial t}=\sum_{i} \vec{\nabla}_{i}\left(-\vec{f}_{i} \mathcal{P}+k_{B} T \vec{\nabla}_{i} \mathcal{P}\right)
$$

where $\overrightarrow{f_{i}}$ is the force on the particle $i$ given by Eq. (9). By introducing the single particle density $\rho(\vec{r}, t) \equiv$ $\left\langle\sum_{i} \delta^{2}\left(\vec{r}-\vec{r}_{i}\right)\right\rangle$, where the average is made over the distribution $\mathcal{P}\left(\vec{r}_{1}, \ldots, \vec{r}_{N}, t\right)$, one obtains,

$$
\begin{aligned}
\mu \frac{\partial \rho}{\partial t}= & -\vec{\nabla}\left[\int d^{2} r^{\prime} \vec{J}\left(\vec{r}-\vec{r}^{\prime}\right) \rho^{(2)}\left(\vec{r}, \vec{r}^{\prime}, t\right)\right. \\
& \left.+\vec{F}^{e}(\vec{r}) \rho(\vec{r}, t)\right]+k_{B} T \nabla^{2} \rho(\vec{r}, t)
\end{aligned}
$$

where $\rho^{(2)}\left(\vec{r}, \vec{r}^{\prime}, t\right)$ is the two-point density. If we now assume that the approximation $\rho^{(2)}\left(\vec{r}, \vec{r}^{\prime}, t\right) \simeq$ $\rho(\vec{r}, t) \rho\left(\vec{r}^{\prime}, t\right)$ is valid, we can then coarse grain the particle-particle interaction force to obtain [6],

$$
\int d^{2} r^{\prime} \vec{J}\left(\vec{r}-\vec{r}^{\prime}\right) \rho\left(\vec{r}^{\prime}, t\right) \simeq-a \vec{\nabla} \rho(\vec{r}, t) ; a \equiv \int d^{2} r \vec{r} \cdot \vec{J}(\vec{r}) / 2,
$$

where only length scales larger than $\lambda$ were considered.

Here we investigate the motion of particles in a twodimensional narrow channel of size $L_{x} \times L_{y}$ under an external force in the $x$-direction, $\vec{F}^{e}=-A(x) \hat{x}$. We then assume that the concentration is only weakly dependent on the transverse $y$-coordinate, $\rho(\mathbf{r}, t) \approx \rho(x, t)$. After collecting all force terms, we obtain,

$$
\mu \frac{\partial \rho}{\partial t}=\frac{\partial}{\partial x}\left\{\rho\left[a \frac{\partial \rho}{\partial x}-A(x)\right]\right\}+k_{B} T \frac{\partial^{2} \rho}{\partial x^{2}} .
$$

Interestingly, by introducing the probability $P(x, t)=$ $\left(L_{y} / N\right) \rho(x, t)$, defining $D=(a N) /\left(\nu L_{y}\right)$, and setting $\mu=1$ and $T=0$, we recover precisely the FPE (5) for $\nu=2$. Herein we restrict our study to a restoring harmonic force, $A(x)=-\alpha x(\alpha \geq 0)$. In the limit $T=0$, the steady-state solution of Eq. (13) is given by

$$
\rho_{s t}=\frac{\alpha}{2 a}\left(x_{e}^{2}-x^{2}\right) ;|x|<x_{e},
$$

with $x_{e}=\left(3 N a / 2 \alpha L_{y}\right)^{1 / 3}$. This result is identical to Eq. (8) if there one adopts $\nu=2$ and $\beta^{*}=x_{e}^{-2}$.

We now show through direct molecular dynamics (MD) simulations that the behavior of a typical overdamped system at $T=0$ is fully compatible with the solution (14), therefore representing a microdynamical realization of Tsallis thermostatistics. As an example, we consider a system of vortex lines moving on a type II superconductor substrate [19, 20], but the results presented here could nevertheless be extended to other forms of shortrange repulsive interactions [8]. In this case, the effective viscosity is given by $\mu=\Phi_{0} H_{c 2} / \omega c^{2}$, where $\Phi_{0}$ is the magnetic quantum flux, $c$ is the speed of light, $\omega$ is the resistivity of the normal phase, and $H_{c 2}$ is the upper critical field. Moreover, the commonly adopted vortex-vortex interaction is $\vec{J}(\vec{r}) \equiv f_{0} K_{1}(|\vec{r}| / \lambda) \hat{r}$, where $K_{1}$ is a modified Bessel function decaying exponentially for $|\vec{r}|>\lambda$, the pre-factor is given by $f_{0}=\Phi_{0}^{2} /\left(8 \pi \lambda^{3}\right)$, and $\lambda$ corresponds to the London penetration length [21].

We perform MD simulations with $N=800$ flux lines placed at random within a two-dimensional channel of sizes $L_{x}=100 \lambda, L_{y}=20 \lambda$, where periodic boundary conditions are imposed in the $y$-direction. In these simulations, we first set $T=0$ and a confining external force is applied in the $x$-direction with restoring constant $\alpha=10^{-3} f_{0} / \lambda$. The equations of motion (9) are numerically integrated and the system evolves in time until a stationary state of mechanical equilibrium is reached, which is identified here in terms of an invariant density profile. As shown in Fig. 1] the obtained stationary profile of particle density is clearly parabolic in shape, in perfect agreement with the theoretical prediction of Eq. (14). 


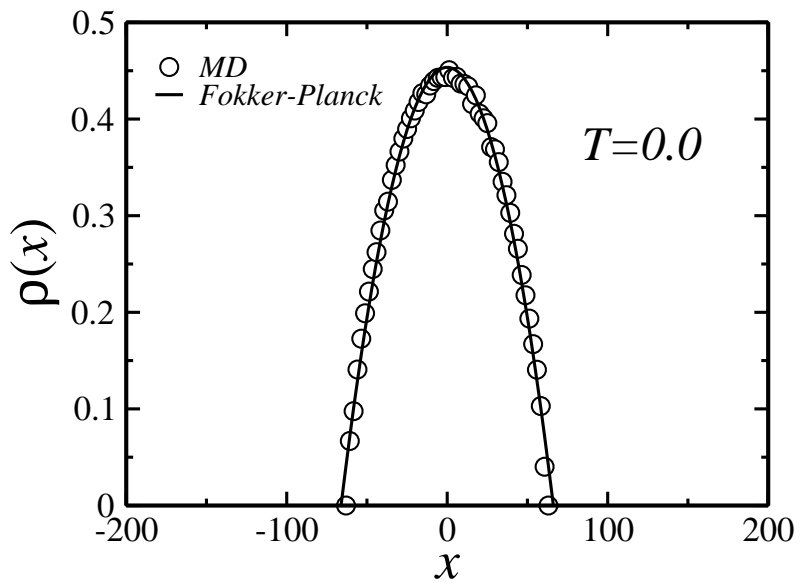

FIG. 1: Profile of the density of particles at stationary state and $T=0$, obtained from molecular dynamics by integrating Eq. (9) (empty circles), as compared to the theoretical estimate Eq. (14). The best fit to the data of a quadratic function gives the parameter $a=2.41 f_{0} \lambda^{3}$ (full line). The position $x$ is measured in units of $\lambda$, whereas the steady-state density $\rho(x)$ is expressed in units $\lambda^{-2}$.

The least-squares fit to the simulation data of a quadratic function results in the parameter $a \approx 2.41 f_{0} \lambda^{3}$. Strictly speaking, the predicted value $a=\pi f_{0} \lambda^{3}$, calculated from Eq. (12), is valid only for unconfined systems, if we assume that the density $\rho$ varies slowly within the interaction range of a particle. The discrete character of the interacting particles therefore leads to a correction on this theoretical prediction. In the absence of external forces, i.e., for $\alpha=0$, our results show that the front propagation at early times evolves as $\langle x(t)\rangle \propto t^{1 / 3}[\underline{6}]$. Once more, the compatibility between this dynamical scaling and the anomalous diffusion behavior intrinsically associated with Eq. (5) for $\nu=2$ confirms the validity of our approach.

Having shown that overdamped particles in the limit of $T=0$, typified as interacting flux lines on a type II superconductor substrate, obeys Tsallis statistics with an entropic index $\nu=2$, we now analyze the effect of finite $T$ on this system. From Eq. (13), one can envisage the competition between two types of diffusion, which are associated, respectively, with the strength of interactions between vortexes, controlled by the parameter, $a$, or equivalently, $D$, and the temperature of the thermal bath, $T$. In this way, the ratio $k_{B} T / a$ plays a crucial role in the time evolution of the system. As for the case $T=0$, in the absence of external forces, i.e., for $\alpha=0$, the diffusion behavior of the system for $k_{B} T \ll a$ should be governed by the anomalous features associated with the index $\nu=2$, namely, $\left\langle x^{2}\right\rangle \propto t^{2 / 3}$, whereas normal diffusion prevails for $k_{B} T \gg a$, i.e., $\left\langle x^{2}\right\rangle \propto t$. In the presence of a restoring external force and for $T>0$, a stationary-state analytical solution for Eq. (13) can still

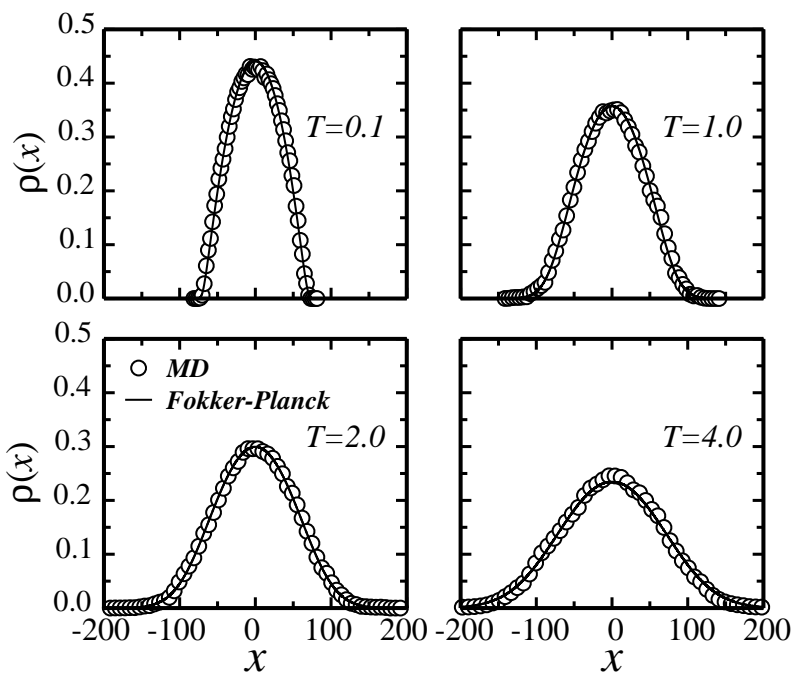

FIG. 2: Comparison between the density of particles profiles in the stationary state obtained from molecular dynamics (empty circles) and the theoretical prediction Eq. (15) (full lines), for different values of the temperature of the thermal bath, $T$. The position $x$ is measured in units of $\lambda$, the density is in units $\lambda^{-2}$, and $T$ is given in units $\left(f_{0} \lambda / k_{B}\right)$. The parameter $a=2.41 f_{0} \lambda^{3}$ is the same adopted for $T=0$, whereas $\rho(0)$ is determined by conservation of the total number of particles in the system, $\int_{-L_{x} / 2}^{L_{x} / 2} \rho(x) d x=\left(N / L_{y}\right)$. For low values of $T$, e.g., $T=0.1 f_{0} \lambda / k_{B}$, the density profile is approximately parabolic. At intermediate values of $T$, the system can be described by a $W$-Lambert function Eq. (15). At high values of $T$, the profile becomes typically Gaussian, as illustrated from results at $T=4 f_{0} \lambda / k_{B}$.

be obtained,

$$
\rho(x)=\frac{k_{B} T}{a} W\left\{\frac{a \rho(0)}{k_{B} T} \exp \left[\frac{a \rho(0)}{k_{B} T}-\frac{\alpha x^{2}}{2 k_{B} T}\right]\right\},
$$

where the $W$-Lambert function is defined implicitly through the equation $W(z) e^{W(z)}=z$ (see 22] and references therein). In order to test this prediction, extensive MD simulations have been performed for different values of $T$. As depicted in Fig. 2, we find very good agreement between the continuum model solution Eq. (15), and MD results of density profiles at steady-state. For consistency, we consider the same numerical value used to adjust the profile at $T=0$, namely, $a=2.41 f_{0} \lambda^{3}$, while the parameter $\rho(0)$ in Eq. (15) has been determined by a conservation constraint in the total number of particles, $\int_{-L_{x} / 2}^{L_{x} / 2} \rho(x) d x=\left(N / L_{y}\right)$.

The effect of increasing $T$ is to gradually change the density profile from a parabolic to a Gaussian shape. Indeed these two limits correspond, respectively, to the particular cases of Tsallis distribution with index $\nu=2$ and the standard Boltzmann-Gibbs statistics. Next we show that the profiles obtained from Eq. (15), for finite $T$, are in fact associated with a novel type of entropy 
form. By comparing Eqs. (13) and (11), we obtain that $\Psi[P(x, t)]=P(x, t)$ and $\Omega[P(x, t)]=2 D P(x, t)+k_{B} T$. Substituting these quantities into Eq. (4), integrating twice and using the conditions (3), one gets,

$$
\begin{aligned}
g[P(x, t)]= & \frac{D}{\bar{\gamma}}\left[P(x, t)-P^{2}(x, t)\right] \\
& -\frac{k_{B} T}{\bar{\gamma}}[P(x, t) \ln P(x, t)],
\end{aligned}
$$

where $\bar{\gamma}$ is a positive Lagrange parameter defined through a free-energy functional like the one given by Eq. (2), and $x$ is a conveniently rescaled variable. This functional leads to the following entropic form:

$$
\begin{aligned}
S[P]= & \frac{D}{\bar{\gamma}}\left[1-\int_{-\infty}^{\infty} d x P^{2}(x, t)\right] \\
& -\frac{k_{B} T}{\bar{\gamma}} \int_{-\infty}^{\infty} d x P(x, t) \ln P(x, t)
\end{aligned}
$$

Equation (17) is precisely the sum of Tsallis entropy with $\nu=2$, which appears as a consequence of many-body interactions, and Boltzmann-Gibbs entropy, which comes from the thermal noise of the bath. The entropy is zero only for $D=T=0$. One may now extremize this mixed entropy under the constraints, $\int_{-\infty}^{\infty} P(x, t) d x=1$ and $U=\int_{-\infty}^{\infty}\left[\phi(x)-\phi_{0}\right] P(x, t) d x$, by defining the functional,

$$
\begin{aligned}
\Sigma[P(x, t)]= & \frac{\bar{\gamma}}{k_{B} T} S[P]-a_{1} \int_{-\infty}^{\infty} d x P(x, t) \\
& -a_{2} \int_{-\infty}^{\infty} d x\left[\phi(x)-\phi_{0}\right] P(x, t)
\end{aligned}
$$

The extreme condition $\delta \Sigma / \delta P=0$ finally leads to

$$
\begin{aligned}
& P(x) \exp \left[\frac{2 D}{k_{B} T} P(x)\right]= \\
& \quad \exp \left\{-\left(1+a_{1}\right)-a_{2}\left[\phi(x)-\phi_{0}\right]\right\},
\end{aligned}
$$

which may be written in terms of $W$-Lambert's function, i.e., in the same form as Eq. (15), by considering a harmonic potential $\phi(x)=\alpha x^{2} / 2$, multiplying both sides by $2 D /\left(k_{B} T\right)$, identifying $a_{2}=1 /\left(k_{B} T\right)$, defining $z$ as the right term in Eq. (19), and $W(z)=2 D P(x) /\left(k_{B} T\right)$. This confirms that the entropy Eq. (17) is directly related to the stationary solution Eq. (15) of the FPE (13).

In summary, we have shown through a nonlinear Fokker-Planck formalism and confirmed through MD simulations that a system of interacting particles undergoing overdamped motion at $T=0$, where $T$ is the temperature of a thermal bath connected to the system, can be considered as a physical realization of Tsallis thermostatistics with an entropic index $\nu=2$ in Eq. (6). At high values of $T$, the classical Boltzmann-Gibbs behavior is recovered. At intermediate values of $T$, our approach, also confirmed by MD simulations, leads to a stationary solution for the corresponding nonlinear FPE that can be expressed in terms of the $W$-Lambert function. As a consequence, we disclose a novel mixed entropic form based on a linear combination between Tsallis and BoltzmannGibbs entropies.

We thank the Brazilian agencies CNPq, CAPES, FAPERJ and FUNCAP, and the CNPq/FUNCAPPronex grant for financial support. We also thank Constantino Tsallis for fruitful discussions.

[1] T. D. Frank, Nonlinear Fokker-Planck Equations: Fundamentals and Applications (Springer, Berlin, 2005).

[2] J. P. Bouchaud and A. Georges, Phys. Rep. 195, 127 (1990).

[3] M. Muskat, The Flow of Homogeneous Fluids Through Porous Media (McGraw-Hill, New York, 1937).

[4] H. Spohn, J. Phys. (France) I 3, 69 (1993).

[5] J. P. Bouchaud et al., J. Phys. (France) II 1, 1465 (1991).

[6] S. Zapperi, A. A. Moreira, and J. S. Andrade, Phys. Rev. Lett. 86, 3622 (2001).

[7] A. A. Moreira et al., Phys. Rev. B 66, 174507 (2002); M. C. Miguel, J. S. Andrade, and S. Zapperi, Braz. J. Phys. 33, 557 (2003); S. Zapperi, J. S. Andrade, and A. A. Moreira, Physica A 342, 383 (2004);

[8] P. Barrozo et al., Phys. Rev. B 80, 104513 (2009).

[9] V. Schwämmle, F. D. Nobre, and E. M. F. Curado, Phys. Rev. E 76, 041123 (2007); V. Schwämmle, E. M. F. Curado, and F. D. Nobre, Eur. Phys. J. B 58, 159 (2007).

[10] M. Shiino, J. Math. Phys. 42, 2540 (2001).

[11] T. D. Frank and A. Daffertshofer, Physica A 295, 455 (2001).

[12] P. H. Chavanis, Phys. Rev. E 68, 036108 (2003).

[13] V. Schwämmle, E. M. F. Curado, and F. D. Nobre, Eur. Phys. J. B 70, 107 (2009).

[14] A. R. Plastino and A. Plastino, Physica A 222, 347 (1995).

[15] C. Tsallis and D. J. Bukman, Phys. Rev. E 54, R2197 (1996).

[16] C. Tsallis, J. Stat. Phys. 52, 479 (1988)

[17] C. Tsallis, Introduction to Nonextensive Statistical Mechanics (Springer, New York, 2009).

[18] E. M. F. Curado and F. D. Nobre, Phys. Rev. E 67, 021107 (2003).

[19] O. Pla and F. Nori, Phys. Rev. Lett. 67, 919 (1991).

[20] V. V. Bryskin and S. N. Dorogotsev, Physica C 215, 173 (1993).

[21] P.-G. de Gennes, Superconductivity of metals and alloys (Benjamin, New York, 1966).

[22] S. R. Valluri et al., J. Math. Phys. 50, 102103 (2009). 